\title{
Coexistence of bicuspid aortic valve, aberrant right subclavian artery and common origin of carotid arteries
}

\author{
P. Tyczyński1 ${ }^{1}$ I. Michałowska² ${ }^{2}$ A. Fronczak ${ }^{3}$, R. Wolny ${ }^{1}$, H. Łazarczyk ${ }^{4}$, S. Kocańda ${ }^{5}$, \\ Z. Chmielak ${ }^{1}$, A. Witkowski ${ }^{1}$ \\ ${ }^{1}$ Department of Interventional Cardiology and Angiology, Institute of Cardiology, Warsaw, Poland \\ ${ }^{2}$ Department of Radiology, Institute of Cardiology, Warsaw, Poland \\ ${ }^{3}$ Department of Arrhythmia, Institute of Cardiology, Warsaw, Poland \\ ${ }^{4}$ Department of Information Technology, Institute of Cardiology, Warsaw, Poland \\ ${ }^{5}$ Department of Cardiac Surgery and Transplantology, Institute of Cardiology, Warsaw, Poland
}

[Received: 2 November 2016; Accepted: 21 January 2017]

\begin{abstract}
Background: Prevalence of bicuspid aortic valve (BAV) and right aberrant subclavian artery (ASA) separately is relatively common in general population, and much higher in some disorders. Surprisingly, coexistence of both valve and vessel anomalies has only been reported in single cases.

Materials and methods: From 2008 to 2016, in a single, high-volume tertiary cardiac centre, patients who underwent chest computed tomography (CT) for various reasons, were retrospectively screened for the presence of right ASA.

Results: Seventy-two patients with either right or left ASA were identified by CT. Among them 7 cases of BAV and right ASA coexistence were identified. Additionally, 1 case with coexisting common origin of carotid arteries (COCA) was visualised in this subgroup.

Conclusions: Although coexistence of ASA and BAV has not been reported in paediatric population, it has been diagnosed in very few adults as well as in our series. Additional presence of COCA in this group seems to be very rare. From practical point of view, heart cannulation via the radial artery and subsequent ASA may be challenging. Similarly, COCA presence may have surgical implications during corrective procedures. (Folia Morphol 2017; 76; 3: 414-419)
\end{abstract}

Key words: bicuspid aortic valve, arteria lusoria, aberrant subclavian artery

\section{INTRODUCTION}

Bicuspid aortic valve (BAV) is a heritable condition, in which the aortic valve has only two leaflets. With this deformity, the valve's function is usually impaired and the entity is associated with significant morbidity including aortic valve stenosis or regurgitation, as well as dilatation, aneurysm, and dissection of the ascending aorta (AA). Based on the fusion pattern of the aortic valve leaflets, different BAV types have been previously described. Some of them (fusion of the right to noncoronary leaflet) are more likely to develop aortopathy. The clinical consequences are the need for periodic assessment of aortic diameters, elective prophylactic surgical aortic repair, and the occurrence of aortic dissection or rupture [10]. However, the decision of how to medically manage BAV-patients or when 
to operate in BAV-associated aortopathy may not be obvious, since aortic dilatation is highly variable and depending on the pre-existing BAV type [18].

Right aberrant subclavian artery (ASA), also known as arteria lusoria, is considered to be rare, however normal variant of the aortic branching. It usually takes-off distal to the left subclavian artery and crosses usually behind the oesophagus to the right upper extremity. Such course of the right ASA may cause a vascular ring around the trachea and oesophagus. Other variants of the right ASA course have also been reported. It may compress the adjacent structures and lead to several symptoms like so called dysphagia lusoria (in case of retro-oesophageal course of ASA), dyspnoea, retrosternal pain or cough.

Prevalence of BAV and right ASA separately is relatively common in general population (1-2\% and $0.1-1 \%$, respectively), and much higher in some disorders, like PHACE syndrome (acronym for Posterior fossa malformations, Haemangiomas, Arterial anomalies, Cardiac defects, Eye abnormalities - a cutaneous condition characterised by multiple congenital abnormalities) [1]. Surprisingly, coexistence of both valve and vessel anomalies has only been reported in single cases (Table 1).

\section{MATERIALS AND METHODS}

From 2008 to 2016, in a single, high-volume tertiary cardiac centre, patients who underwent chest computed tomography (CT) for various reasons, were retrospectively screened for the presence of right ASA (Figs. 1-7).

Type of BAV has been assessed by both CT and echocardiography. Two classifications have been used. Classification by Schaefer et al. [15] is traditional and based on fused two cusps and the orientation of the raphe; type 1 - fusion of right and left coronary cusp; type 2 - right and non-coronary fusion; and type 3 - left and non-coronary fusion. Classification by Kang et al. [6] is based on the BAV orientation only. Two BAV phenotypes - fusion of the right and left coronary cusps (BAV-AP) and fusion of the right or left coronary cusp and non-coronary cusp - are used.

\section{RESULTS}

Seventy-two patients with either right or left ASA were identified. In 7 patients right ASA was diagnosed by CT.

Demographics, comorbidities, history of previous interventions and current corrective procedures are presented in Table 2. The identification of BAV and ASA coexistence took place in the adulthood of all but 1 patient (17 years old). Nonetheless, some of
Table 1. Reported coexistence of bicuspid aortic valve and aberrant right subclavian artery

\begin{tabular}{ll}
\hline Authors & Predominant clinical picture \\
\hline Angeli et al. & Turner syndrome, right-sided vena cava superior \\
Asano et al. & Coarctation of aorta \\
Jacobsen et al. & $\begin{array}{l}\text { Ventricular septal defect, interruption } \\
\text { of the aortic arch }\end{array}$ \\
Kono et al. & Subclavian artery aneurysm \\
Marinho et al. & Subclavian artery aneurysm \\
Pavlidis et al. & Ectopic left vertebral artery \\
\hline
\end{tabular}

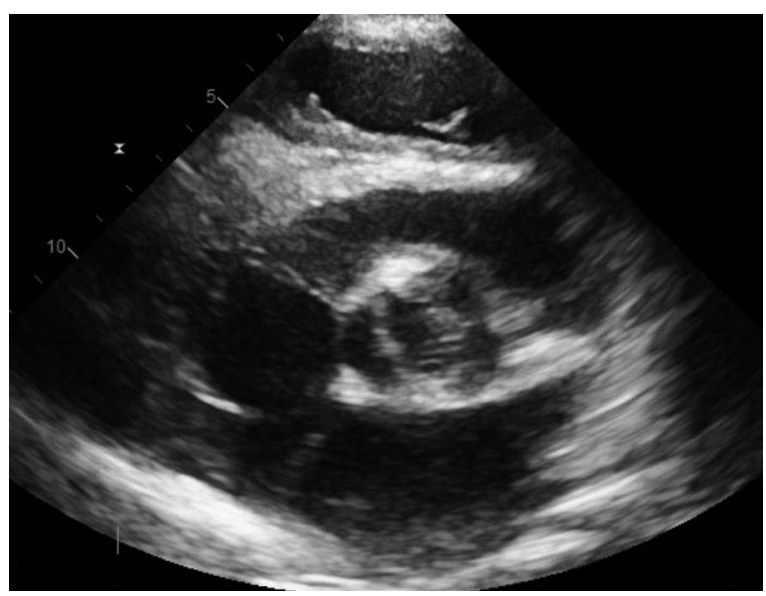

Figure 1. Patient 1. Echocardiography — parasternal short axis image at the aortic valve level. Bicuspid aortic valve in systolic frame with fusion of right and left coronary cusps.

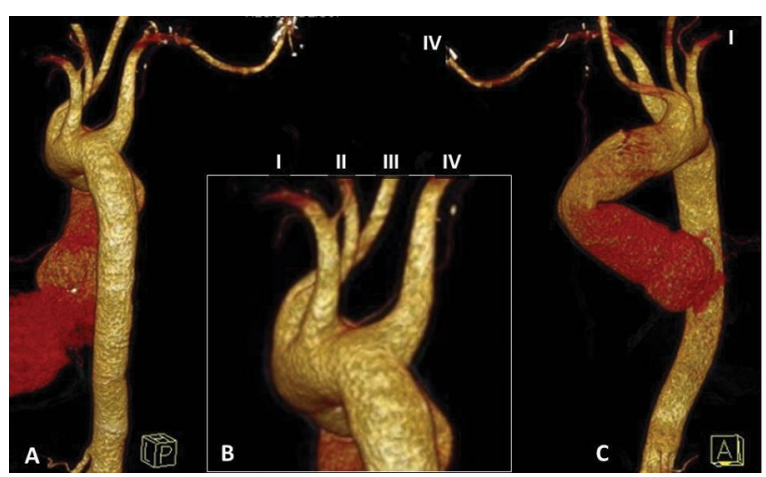

Figure 2. Patient 1 . Computed tomography angiography of the thoracic aorta, volume rendered reconstruction; A. Postero-lateral view; B. Magnification of the image "A" at the level of the aortic arch: I - left subclavian artery, II — left common carotid artery, III — right common carotid artery, IV — right aberrant subclavian artery; C. Anterior view.

the patients had a history of corrective procedures in their childhood. 


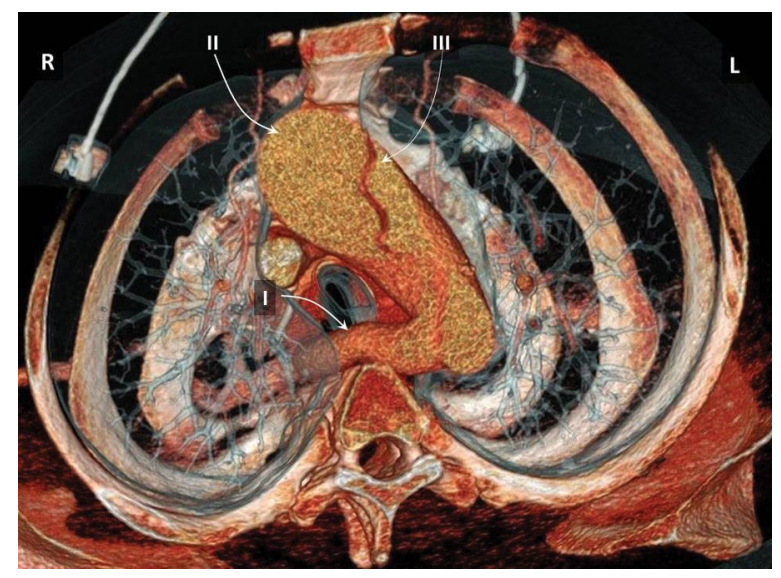

Figure 3. Patient 2. Computed tomography angiography, volume rendered reconstruction of the aortic arch from inferior view; I right aberrant subclavian artery originating from the descending segment of the aortic arch, II - ascending segment of the aortic arch, III — aortic dissection; $\mathrm{R}$ - right side; $\mathrm{L}$ — left side.

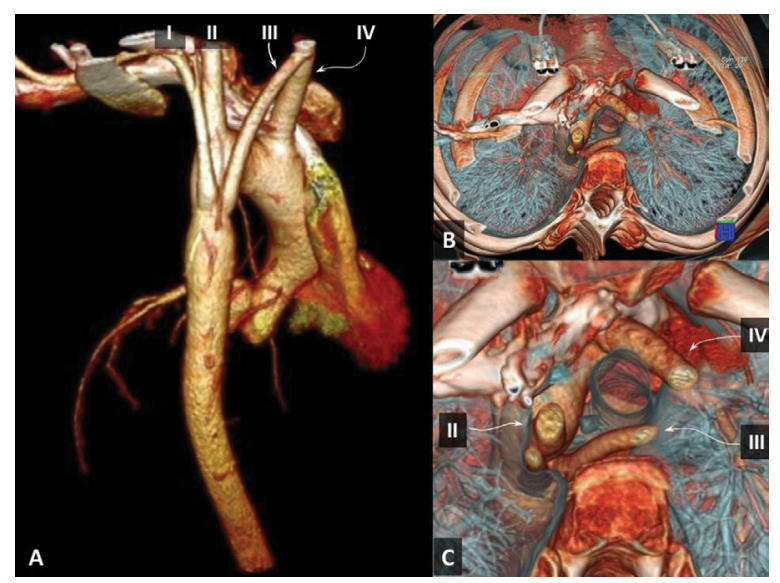

Figure 4. Patient 4. Computed tomography angiography, volume rendered reconstruction of the aorta; $\mathbf{A}$. Branches of the aortic arch from the posterior view: I — left subclavian artery, II — left common carotid artery, III — right aberrant subclavian artery, IV — right common carotid artery; B. Branches of the aortic arch from superior view; C. Magnification of the image " $B$ " with focus on the aortic arch branches. Retrotracheal course of the right aberrant subclavian artery.

Types of BAV are presented in Table 3. In most of the cases the right and left leaflet are fused.

In all patients the ASA course was behind the trachea and oesophagus.

\section{DISCUSSION}

More than 20 aortic arch configurations have been described, among them both right and left ASA. Embryologically, development of the aortic arch and its branches is a complex process and resulting from the formation and selective involution of paired vascular

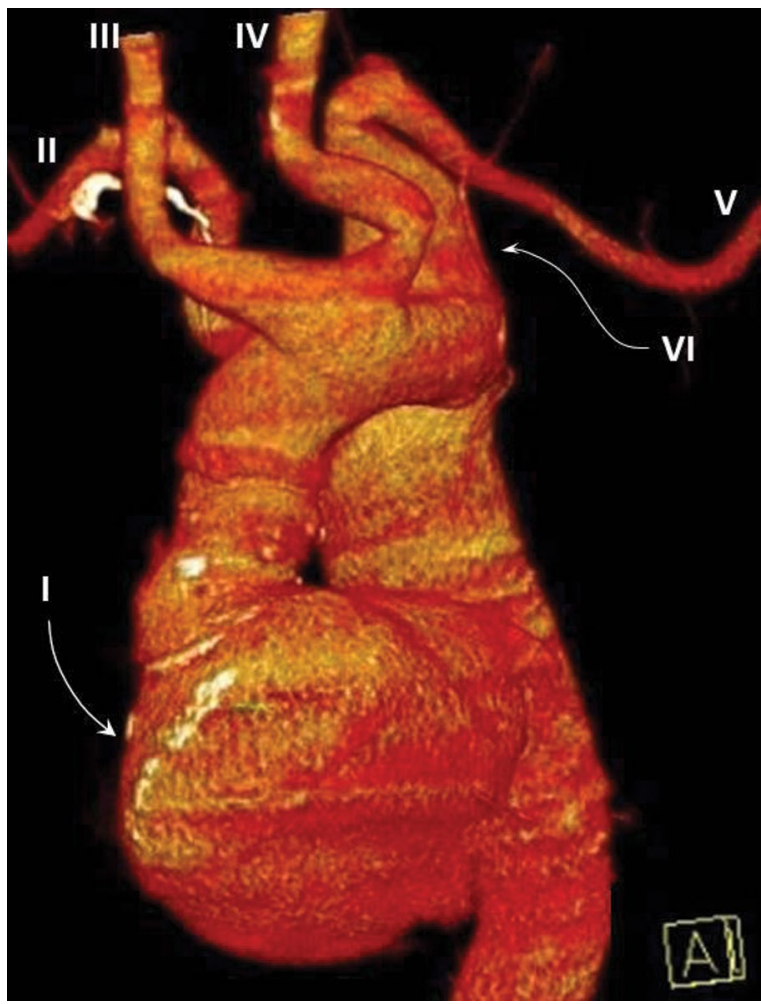

Figure 5. Patient 5. Computed tomography angiography, volume rendered reconstruction of the thoracic aorta from anterior view; I - aneurysm of the Valsalva sinus, II - right aberrant subclavian artery, III and IV — right and left common carotid arteries, respectively, with their common origin, $\mathrm{V}$ — left subclavian artery, $\mathrm{VI}$ dilated origin of the left subclavian artery.

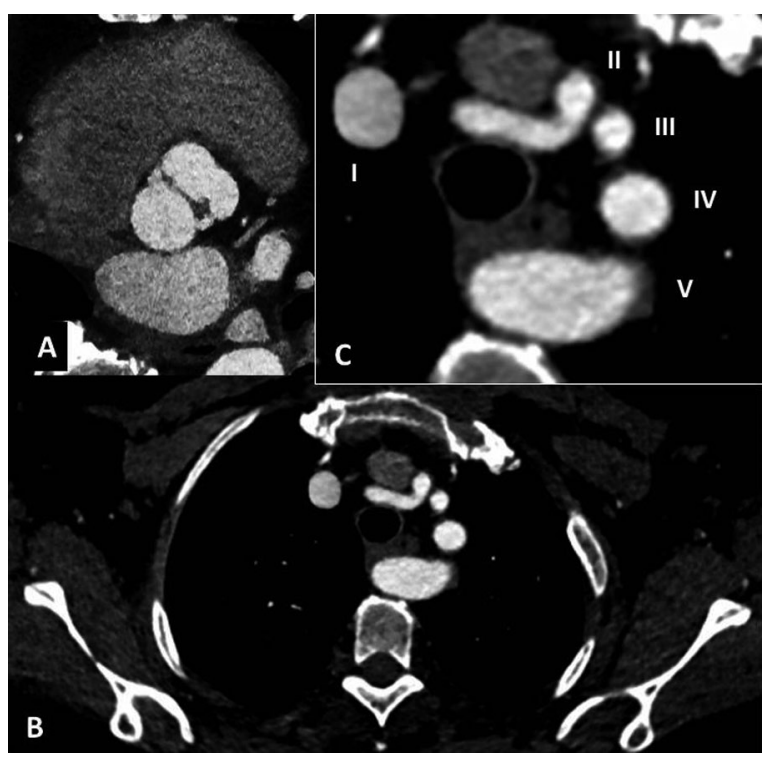

Figure 6. Patient 6. Multiplanar reformatted computed tomography images; A. Bicuspid aortic valve; B. Cross-section of the large vessels; C. Magnification of the image "B": I - superior vena cava, II — right common carotid artery, III — left common carotid artery, IV - left subclavian artery, V - right aberrant subclavian artery. 


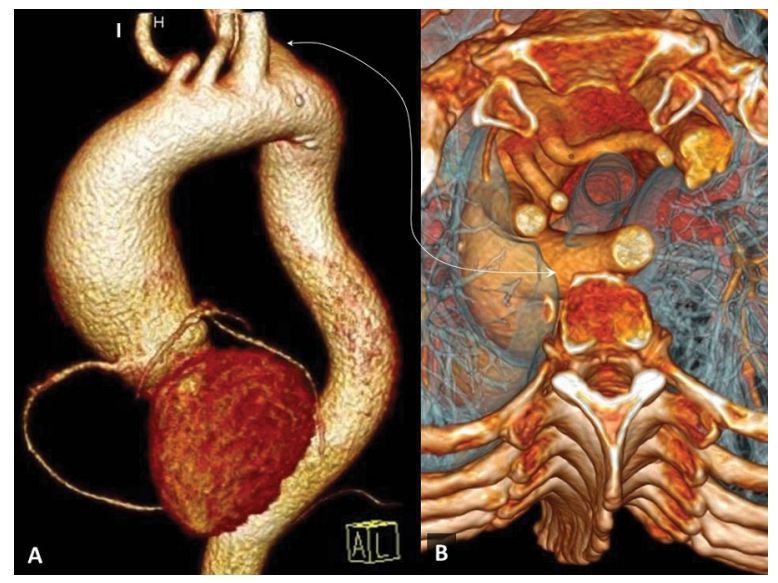

Figure 7. Patient 6 . Computed tomography angiography, volume rendered reconstruction of the aortic arch; A. Antero-lateral view; I - right common carotid artery; B. Superior view. Visible retrotracheal course of the right aberrant subclavian artery. Double-headed arrow indicates the right aberrant subclavian artery. arches connecting the embryonic aortic sac (ventral aorta) with paired dorsal aortae and is described in detail elsewhere [7]. Briefly, proximal segments of the third pair of the primitive aorta form the common carotid arteries and the right fourth arch forms the proximal right subclavian artery. Right ASA occurs when there is abnormal obliteration of the right fourth vascular arch and the portion of the right dorsal aorta cranial to the seventh intersegmental artery [3].

In an impressive study of more than 15,000 initial paediatric echocardiograms, ASA was identified in 226 patients (171 with left aortic arch), of whom only $30 \%$ had normal intracardiac anatomy. Most common ASA-associated anomalies were conotruncal defects (especially tetralogy of Fallot) [14]. No BAV was reported here.

In another large study of approximately 11,000 pathological specimens, ASA was identified in 128

Table 2. Demographics and coexisting anomalies

\begin{tabular}{|c|c|c|c|c|c|}
\hline Patient & $\begin{array}{c}\text { Age } \\
\text { [years] }\end{array}$ & Sex & Clinical presentation & Previous medical history & Corrective procedures \\
\hline 1 (Figs. 1, 2) & 42 & $\mathrm{~F}$ & Acute AA dissection & - & AA replacement \\
\hline 2 (Fig. 3) & 40 & M & Acute AA dissection & AA aneurysm, HT, IS & AA replacement \\
\hline 3 & 47 & M & $\mathrm{AA}$ aneurysm & End-stage renal disease & $\begin{array}{l}\text { AA aneurysm resection with } \mathrm{RCL} \\
\text { correction and subsequent AVR }\end{array}$ \\
\hline 4 (Fig. 4) & 17 & $\mathrm{~F}$ & $\begin{array}{l}\text { Tunnel LVOT obstruction and } \\
\text { hypoplastic AA }\end{array}$ & $\begin{array}{l}\text { Subaortic membrane resection and correc- } \\
\text { tion of descending CoA at the age of } 10 \text {. } \\
\text { Re-operation for recurrence of subaortic } \\
\text { stenosis at the age of } 12\end{array}$ & $\begin{array}{l}\text { Konno procedure with AVR } \\
\text { and subsequent pacemaker } \\
\text { implantation }\end{array}$ \\
\hline 5 (Fig. 5) & 34 & $\mathrm{~F}$ & CoA & $\begin{array}{l}\text { Turner syndrome, } A F, A A \text { aneurysm } \\
\text { operation several years ago }\end{array}$ & $\begin{array}{l}\text { Resection of the aortic root } \\
\text { aneurysm and AVR }\end{array}$ \\
\hline 6 (Figs. 6, 7) & 61 & $\mathrm{~F}$ & Non-specific chest pain & $H T$, mild $A A$ aneurysm & Exclusion of $A A$ dissection \\
\hline 7 (Fig. 8) & 78 & M & $\begin{array}{l}\text { Moderate AS, } \\
\text { AA aneurysm }\end{array}$ & - & - \\
\hline
\end{tabular}

AA — ascending aorta; AF — atrial fibrillation; AS — aortic stenosis; AVR — aortic valve replacement; CoA — coarctation of the aorta; F — female; HT — hypertension; IS — ischaemic stroke; LVOT — left ventricular outflow tract; $\mathrm{M}$ - male; $\mathrm{RCL}$ - right coronary leaflet of the aortic valve

Table 3. Type of bicuspid aortic valve and coexisting valve anomalies and the course of the right aberrant subclavian artery

\begin{tabular}{lcccc}
\hline $\begin{array}{l}\text { Patient } \\
\text { number }\end{array}$ & Classification by Schaefer & Type of BAV & $\begin{array}{c}\text { Other arch } \\
\text { anomalies }\end{array}$ \\
\cline { 2 - 5 } & Right-left (1) & Classification by Kang & - \\
2 & Right-left (1) & (1) & $(1)$ & - \\
3 & & & $(2)$ & - \\
4 & Non-coronary-right (2) & & & COCA \\
5 & & Undetermined* & $(1)$ & - \\
7
\end{tabular}

*Confirmed intraoperatively; BAV — bicuspid aortic valve; COCA — common origin of the carotid arteries 


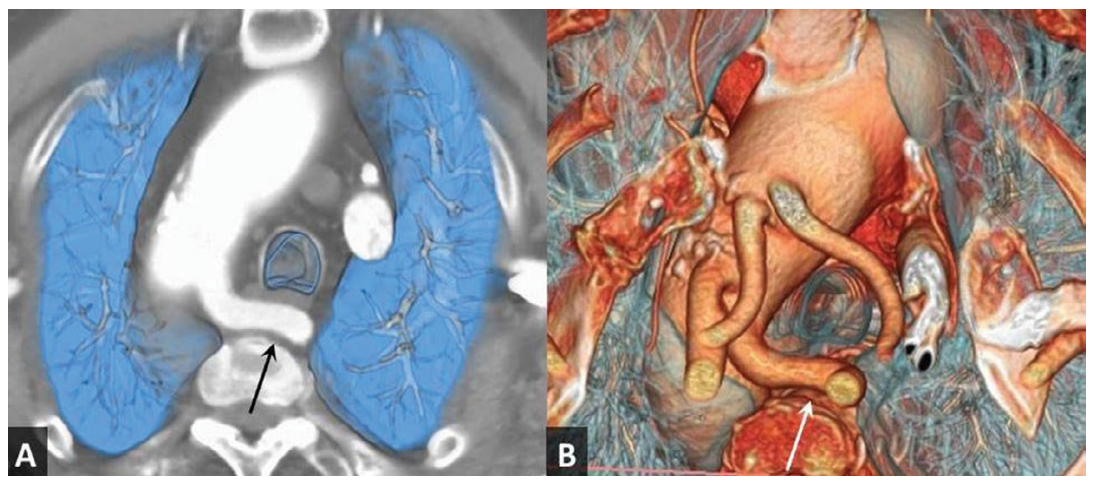

Figure 8. Patient 7. Computed tomography angiography; A. Multiplanar reformatted image with cross-section of the superior pulmonary lobes and trachea (blue colour) and the aortic arch; $\mathbf{B}$. Volume rendered reconstruction of the aortic arch at the same level. Arrows indicate the retrotracheal and retro-oesophageal course of the right aberrant subclavian artery.

patients, among whom 117 patients with a congenital heart disease [20]. Comparable to previous study, most frequently diagnosed conditions were truncus arteriosus and left heart obstruction. In none of them BAV was identified.

Notably both of these two studies had some significant limitations. Many of the conditions found in the pathological specimens were lethal, while BAV is not a lethal disorder. Furthermore, these studies included predominantly paediatric populations as opposite to adult patients in our series.

Different courses of the right ASA have been described. Right ASA courses to the right behind the oesophagus in majority of the cases (80\%), between the oesophagus and trachea in 15\%, and anterior to the trachea or mainstem bronchus in 5\% [5]. Thus, our group presented typical ASA course.

Bicuspid aortic valve has been extensively described elsewhere. Prevalence may be much higher in some disorders, like Turner syndrome [11]. In this group of 99 adult women the most common BAV-associated anomaly was coarctation of aorta (46\%). Such high association (32\%) of BAV and coarctation of aorta was also observed in the group of 229 paediatric BAV-patients [9]. Among other corrective procedures of these children, surgical repair of coarctation of aorta and subaortic membrane resection were required in $24 \%$ and nearly $5 \%$, respectively. During follow-up AA dilatation was observed in $33 \%$ of patients. Recurrence of subaortic stenosis after surgical correction is frequent. In a study by Valeske et al. [17] 31\% patients needed reoperation due to left ventricular outflow tract restenosis. All this is roughly in line with our observations.

BAV types. In our series type 1 BAV were present in majority of patients, what is in line with observa- tions from large cohorts. In study by Schafer et al. [15], type 2 BAV (right and non-coronary leaflet fusion) was associated with AA dilatation. Nonetheless in our patient with type 2 BAV (no. 5), hypoplastic AA was observed.

Common origin of carotid arteries (COCA), also referred to as common carotid trunk, results from persistence of the foetal common carotid trunk [13]. It has only been reported in single adults cases mostly in coexistence with right ASA $[2,4,8,12,16]$, but also with other congenital cardiovascular lesions [19]. To our best knowledge coexistence of BAV, ASA and COCA has not been reported so far.

\section{CONCLUSIONS}

Coexistence of ASA and BAV has not been reported in paediatric population, but only in very few adults. Our series elucidates clinical characteristics of adult patients with such coexistence of anomalies. Additional presence of COCA in this group seems to be very rare.

From practical point of view, heart cannulation via the radial artery and subsequent ASA may be challenging. Awareness of ASA presence is also important during work-up of oesophageal operations. Thus, ASA-patients are at higher risk of iatrogenic injuries during radiological or surgical interventions. Similarly, COCA presence may have surgical implications during corrective procedures [13].

\section{WHAT IS KNOWN ABOUT THE TOPIC}

Characteristics of BAV and ASA separately are quite well known. However, coexistence of BAV and right ASA has only been reported in single cases, mostly in the paediatric patients. Along with increas- 
ing population of adults with corrected congenital heart diseases, there is a need for systematic studies in this population. To the best of our knowledge, there has been no previous report on the coexistence of BAV, ASA and COCA.

\section{WHAT DOES THIS STUDY ADD}

Our report elucidates comprehensively clinical characteristics of a series of adult patients with rare presence of both BAV and ASA. This is also the first report of BAV, ASA and COCA coexistence. Since such anomalies often occur in patients with concomitant congenital disorders of heart and great vessels, the need for surgical corrections is not infrequent in this population. As the presence of additional vascular anomalies may significantly complicate the procedure, detailed preoperative evaluation by imaging modalities is crucial for reducing the risk of intervention. What is more, heart cannulation via the radial artery and subsequent ASA may be challenging.

\section{REFERENCES}

1. Bayer ML, Frommelt PC, Blei F, et al. Congenital cardiac, aortic arch, and vascular bed anomalies in PHACE syndrome (from the International PHACE Syndrome Registry). Am J Cardiol. 2013; 112(12): 1948-1952, doi: 10.1016/j.amjcard.2013.08.025, indexed in Pubmed: 24079520.

2. Chahwan S, Miller MT, Kim KA, et al. Aberrant right subclavian artery associated with a common origin of carotid arteries. Ann Vasc Surg. 2006; 20(6): 809-812, doi: 10.1007/ s10016-006-9074-3, indexed in Pubmed: 16779507.

3 http://radiologykey.com/.

4. Jan SL, Hwang B, Fu YC, et al. A rare vascular ring caused by an aberrant right subclavian artery concomitant with the common carotid trunk: a report of two cases. Int J Cardiovasc Imaging. 2006; 22(2): 253-256, doi: 10.1007/ s10554-005-9008-0, indexed in Pubmed: 16049622.

5. Kadir S. Regional anatomy of the thoracic aorta. In: Atlas of Normal and Variant Angiographic Anatomy, S. Kadir, Ed., WB Saunders, Philadelphia, Pa, USA 1991.

6. Kang JW, Song HG, Yang DH, et al. Association between bicuspid aortic valve phenotype and patterns of valvular dysfunction and bicuspid aortopathy: comprehensive evaluation using MDCT and echocardiography. JACC Cardiovasc Imaging. 2013; 6(2): 150-161, doi: 10.1016/j. jcmg.2012.11.007, indexed in Pubmed: 23489528.

7. Kau T, Sinzig M, Gasser J, et al. Aortic development and anomalies. Semin Intervent Radiol. 2007; 24(2): 141-152, doi: 10.1055/s-2007-980040, indexed in Pubmed: 21326792.

8. Kokotsakis I, Harling L, Anagnostakou V, et al. Single-stage surgical repair in a complex case of aberrant right subclavian artery aneurysm and common carotid trunk. J Cardiothorac Surg. 2013; 8: 112, doi: 10.1186/1749-8090-8-112, indexed in Pubmed: 23618166.

9. Malčić I, Grgat J, Kniewald H, et al. [Bicuspid aortic valve and left ventricular outflow tract defects in ciiildren - syndrome of bicuspid aortopathy?]. Lijec Vjesn. 2015; 137(910): 267-275, indexed in Pubmed: 26749948.

10. Michelena HI, Della Corte A, Prakash SK, et al. Bicuspid aortic valve aortopathy in adults: Incidence, etiology, and clinical significance. Int J Cardiol. 2015; 201: 400-407, doi: 10.1016/j. ijcard.2015.08.106, indexed in Pubmed: 26310986.

11. Mortensen $\mathrm{KH}$, Hjerrild BE, Andersen NH, et al. Abnormalities of the major intrathoracic arteries in Turner syndrome as revealed by magnetic resonance imaging. Cardiol Young. 2010; 20(2): 191-200, doi: 10.1017/ S1047951110000041, indexed in Pubmed: 20307329.

12. Murzi M, Mariani M, Tiwari KK, et al. Aberrant right subclavian artery aneurysm in coexistence with a common carotid trunk. Ann Thorac Surg. 2009; 88(1): e8, doi: 10.1016/j. athoracsur.2009.04.110, indexed in Pubmed: 19559178.

13. Poultsides GA, Lolis ED, Vasquez J, et al. Common origins of carotid and subclavian arterial systems: report of a rare aortic arch variant. Ann Vasc Surg. 2004; 18(5): 597-600, doi: 10.1007/ s10016-004-0060-3, indexed in Pubmed: 15534741.

14. Ramaswamy P, Lytrivi ID, Thanjan MT, et al. Frequency of aberrant subclavian artery, arch laterality, and associated intracardiac anomalies detected by echocardiography. Am J Cardiol. 2008; 101(5): 677-682, doi: 10.1016/j.amjcard.2007.10.036, indexed in Pubmed: 18308020 .

15. Schaefer BM, Lewin MB, Stout KK, et al. The bicuspid aortic valve: an integrated phenotypic classification of leaflet morphology and aortic root shape. Heart. 2008; 94(12): 1634-1638, doi: 10.1136/hrt.2007.132092, indexed in Pubmed: 18308868.

16. Tsai IC, Tzeng WS, Lee T, et al. Vertebral and carotid artery anomalies in patients with aberrant right subclavian arteries. Pediatr Radiol. 2007; 37(10): 1007-1012, doi: 10.1007/ s00247-007-0574-2, indexed in Pubmed: 17768615.

17. Valeske $K$, Huber $C$, Mueller M, et al. The dilemma of subaortic stenosis--a single center experience of 15 years with a review of the literature. Thorac Cardiovasc Surg. 2011; 59(5): 293-297, doi: 10.1055/s-0030-1271039, indexed in Pubmed: 21544788.

18. Wang $Y$, Wu B, Li J, et al. Impact of aortic insufficiency on ascending aortic dilatation and adverse aortic events after isolated aortic valve replacement in patients with a bicuspid aortic valve. Ann Thorac Surg. 2016; 101(5): 1707-1714, doi: 10.1016/j.athoracsur.2015.10.047, indexed in Pubmed: 26794893.

19. Wells TR, Landing BH, Shankle WR. Syndromal associations of common origin of the carotid arteries. Pediatr Pathol. 1993; 13(2): 203-212, indexed in Pubmed: 8464781.

20. Zapata H, Edwards JE, Titus JL. Aberrant right subclavian artery with left aortic arch: associated cardiac anomalies. Pediatr Cardiol. 1993; 14(3): 159-161, doi: 10.1007/ BF00795645, indexed in Pubmed: 8415218. 